Fatou and brothers Riesz theorems in the infinite-dimensional polydisc

\author{
Aleman, Alexandru
}

2019-03

Aleman , A , Olsen , J-F \& Saksman , E 2019 , ' Fatou and brothers Riesz theorems in the infinite-dimensional polydisc ' , Journal d'Analyse Mathematique , vol. 137 , no. 1 , pp.

429-447 . https://doi.org/10.1007/s11854-019-0006-x

http://hdl.handle.net/10138/312569

https://doi.org/10.1007/s11854-019-0006-x

cc_by_nc_sa

acceptedVersion

Downloaded from Helda, University of Helsinki institutional repository.

This is an electronic reprint of the original article.

This reprint may differ from the original in pagination and typographic detail.

Please cite the original version. 


\title{
FATOU AND BROTHERS RIESZ THEOREMS IN THE INFINITE-DIMENSIONAL POLYDISC
}

\author{
ALEXANDRU ALEMAN, JAN-FREDRIK OLSEN, AND EERO SAKSMAN
}

\begin{abstract}
We study the boundary behavior of functions in the Hardy spaces on the infinite dimensional polydisk. These spaces are intimately related to the Hardy spaces of Dirichlet series. We exhibit several Fatou and MarcinkiewiczZygmund type theorems for radial convergence. As a consequence one obtains easy new proofs of the brothers F. and M. Riesz Theorems in infinite dimensions, as well as being able to extend a result of Rudin concerning which functions are equal to the modulus of an $H^{1}$ function almost everywhere to $\mathbb{T}^{\infty}$. Finally, we provide counterexamples showing that the pointwise Fatou theorem is not true in infinite dimensions without restrictions to the mode of radial convergence even for bounded analytic functions.
\end{abstract}

\section{INTRODUCTION}

The object of study in this paper is the Hardy spaces $H^{p}$ on the infinite dimensional torus $\mathbb{T}^{\infty}=\left\{\left(z_{1}, z_{2}, \ldots\right): z_{n} \in \mathbb{T}\right\}$. In recent years, there has been a renewed interest in these spaces, mainly due to their connection to Dirichlet series and thereby to analytic number theory. We refer to [9] for the related theory of Dirichlet series and for basic references to the field.

In order to recall the definition of the space $H^{p}\left(\mathbb{T}^{\infty}\right)$ for $p \in[1, \infty]$, observe that $\mathbb{T}^{\infty}$ is a compact abelian group with dual $\mathbb{Z}^{\infty}$ and Haar measure $\mathrm{d} \theta=\mathrm{d} \theta_{1} \times \mathrm{d} \theta_{2} \times$ $\cdots$, where $\mathrm{d} \theta_{n}$ is the normalised Haar measure on the $n$-th copy of $\mathbb{T}$. Elements $f$ in $L^{p}\left(\mathbb{T}^{\infty}\right)$ are uniquely defined by their Fourier series expansion (see, e.g., [10])

$$
f \sim \sum_{\nu \in \mathbb{Z}_{0}^{\infty}} a_{\nu} \mathrm{e}^{\mathrm{i} \theta \cdot \nu}
$$

where the Fourier coefficients are defined in the standard manner and $\nu \in \mathbb{Z}_{0}^{\infty}$ means that only finitely many of the components of the index sequence $\nu$ are nonzero. One may now define the Hardy spaces $H^{p}$ to be the analytic part of $L^{p}$ in the following way

$$
H^{p}\left(\mathbb{T}^{\infty}\right)=\left\{f \in L^{p}\left(\mathbb{T}^{\infty}\right): f \sim \sum_{\nu \in \mathbb{N}_{0}^{\infty}} a_{\nu} \mathrm{e}^{\mathrm{i} \theta \cdot \nu}\right\} .
$$

2000 Mathematics Subject Classification. 32A05, 32A40, 31A20,42B30.

Key words and phrases. Boundary behaviour, infinite polydisc, Fatou type theorems, brothers Riesz theorems.

The third author was supported by the Finnish Academy CoE in Analysis and Dynamics Research and by a Knut and Alice Wallenberg Grant. 
Note that also other notions of analyticity are possible in this setting (see, e.g., our Corollary [5]).

A basic, and extremely useful, feature of the one variable theory is that any function $f \in H^{p}(\mathbb{T})$ can be extended to an analytic function on the open unit disc $\mathbb{D}$. In particular, the function $\mathrm{e}^{\mathrm{i} t} \longmapsto f\left(r \mathrm{e}^{\mathrm{i} t}\right)$ is smooth and approximates the function $f$ in norm as $r \nearrow 1$, (weak- $*$, if $p=\infty$ ) and, for almost every $\mathrm{e}^{\mathrm{i} t} \in \mathbb{T}$, it holds that $f\left(r e^{\mathrm{i} t}\right) \rightarrow f\left(e^{\mathrm{i} t}\right)$. This remains true in finite dimensions with almost no restrictions to the radial (or even non-tangential) approach, see Remark 4 and Corollary 4 below.

The purpose of the current paper is to initiate the investigation of to which extent such Fatou-type approximations hold in the infinite dimensional setting. We note that [12] contains some first steps in this direction for the space $H^{\infty}\left(\mathbb{T}^{\infty}\right)$.

While $\mathbb{T}^{\infty}$ is the distinguished boundary of $\left.\mathbb{D}^{\infty}=\left(z_{1}, z_{2}, \ldots\right): z_{n} \in \mathbb{D}\right\}$, it is no longer straight-forward to extend functions $f \in H^{p}\left(\mathbb{T}^{\infty}\right)$ to functions on the polydisc $\mathbb{D}^{\infty}$. This is because point evaluations for Hardy functions in the polydisk are well-defined only in $\ell^{2} \cap \mathbb{D}^{\infty}$ for $p<\infty$, see [2], and in $c_{0} \cap \mathbb{D}^{\infty}$ for $p=\infty$, see [6]. In particular, when formulating Fatou-type results, these restrictions have to be kept in mind.

Our first result, Theorem 1 below, considers a boundary approach of the type

$$
\left(r e^{i \theta_{1}}, r^{2} e^{i \theta_{2}}, r^{3} e^{i \theta_{3}}, \ldots\right) \quad \text { with } r \nearrow 1^{-},
$$

and shows that the standard Fatou type results remain valid for functions with Fourier spectrum supported on $\mathbb{N}_{0}^{\infty} \cup\left(-\mathbb{N}_{0}^{\infty}\right)$. As a corollary, one obtains easy proofs of infinite dimensional versions of some results due to the brothers $\mathrm{M}$. and F. Riesz, see Corollaries 1 and 2. Corollary 3 yields a useful characterisation of elements $f \in H^{1}\left(\mathbb{T}^{\infty}\right)$ in terms of uniform $L^{1}$-boundedness of their the 'mte Abschnitt'. Theorem 2 generalizes to infinite dimensions the theorem of Marcinkiewicz and Zygmund concerning the vanishing radial limits of singular measures that have Fourier series supported on $\mathbb{N}_{0}^{\infty} \cup\left(-\mathbb{N}_{0}^{\infty}\right)$. Finally, Section 3 provides counter examples to unrestricted radial approach in infinite dimensions and poses some open questions. In particular, Theorem 4 yields a bounded analytic function with no boundary limit at almost every boundary point for a suitable radial approach.

\section{Fatou And Brother Riesz theorems in $\mathbb{T}^{\infty}$}

The inspiration for the present paper, as well as parts of the above-cited paper [12, is the work of Helson [4. He introduced so-called vertical limit functions to the theory of Dirichlet series in order to extend them analytically up to the imaginary axis. Somewhat simplified, he showed that a Dirichlet series

$$
F(s)=\sum_{n=1}^{\infty} a_{n} n^{-s},
$$


for which $\left(a_{n}\right)_{n=1}^{\infty} \in \ell^{2}$ can, in a weak sense, be extended to all of Re $s>0$. This is not immediately clear, since such Dirichlet series, in general, will only converge when Re $s>1 / 2$ (this follows, e.g., from Cauchy-Schwarz). However, the effect of taking vertical limits of $F(s)$ is to replace the coefficients $\left(a_{n}\right)_{n=1}^{\infty}$ by $\left(\chi(n) a_{n}\right)_{n=1}^{\infty}$, where $n \longmapsto \chi(n)$ is a function from $\mathbb{N}$ to $\mathbb{T}$ that is multiplicative in the sense that $\chi(n m)=\chi(n) \chi(m)$ for all $n, m \in \mathbb{Z}$. The statement of Helson is essentially that for almost every choice of $\chi$, the modified Dirichlet series, which we may denote by $F_{\chi}$, has an analytic extension up to the imaginary axis. Helson's proof combines Fubini with one variable results to analytically extend $F_{\chi}(\mathrm{it})$ to the right-half plane.

The relation of Helson's result to Hardy spaces $H^{p}\left(\mathbb{T}^{\infty}\right)$ takes place through the fundamental connection between Dirichlet series and Hardy spaces on the polydisc, due to H. Bohr [1]. Indeed, functions on $\mathbb{T}^{\infty}$ formally become Dirichlet series when restricted to the path $t \mapsto\left(p_{n}^{-\mathrm{i} t}\right)$, where $p_{n}$ is the $n$-th prime number. Explicitly,

$$
f \sim \sum_{\nu \in \mathbb{N}_{0}^{\infty}} a_{\nu} \mathrm{e}^{\mathrm{i} \theta \cdot \nu} \Longrightarrow F(\mathrm{i} t):=f\left(p_{1}^{\mathrm{i} t}, p_{2}^{\mathrm{i} t}, \ldots\right) \sim \sum_{n=1}^{\infty} a_{n} n^{-\mathrm{i} t}
$$

where $n=p_{1}^{\nu_{1}} \cdots p_{k}^{\nu_{k}}$ and we identify $a_{\nu}$ to the corresponding coefficient $a_{n}$. Also note that for $\sigma>1 / 2$, the slightly modified path $t \mapsto\left(p_{n}^{-i t-\sigma}\right)$ lies in $\mathbb{D}^{\infty} \cap \ell^{2}$, and so, by the above mentioned results on bounded point evaluations, every $f \in$ $H^{p}\left(\mathbb{T}^{\infty}\right)$ restricts to an analytic Dirichlet series. These restrictions exactly form the Dirichlet-Hardy spaces $\mathscr{H}^{p}$. Helson's result can then be reformulated as follows: For almost every $\chi \in \mathbb{T}^{\infty}$, the restriction of a function $f \in H^{p}\left(\mathbb{T}^{\infty}\right)$ to the path $t \mapsto\left(\chi\left(p_{n}\right) p_{n}^{-i t-\sigma}\right)$ gives an analytic function on $\operatorname{Re}(\sigma+\mathrm{i} t)=\sigma>0$.

A similar scheme was used in [12] to approximate functions $f \in H^{\infty}\left(\mathbb{T}^{\infty}\right)$ almost everywhere. First, note that the restriction $F(s)$ is analytic on $\mathbb{C}_{+}=\{\operatorname{Re} s>0\}$, due to [6] and [1] (also, in this connection see [8]). Next, fix $\chi=\mathrm{e}^{\mathrm{i} \theta_{0}}$, and extend $\theta \mapsto f_{\mathrm{e}^{\mathrm{i}} \theta_{0}}\left(\mathrm{e}^{\mathrm{i} \theta}\right):=f\left(\mathrm{e}^{\mathrm{i}\left(\theta+\theta_{0}\right)}\right)$ to the analytic function $F_{\mathrm{e}^{\mathrm{i} \theta_{0}}}(s)$. Reversing the roles of the variables, put $\tilde{f}_{s}\left(\mathrm{e}^{\mathrm{i} \theta_{0}}\right):=F_{\mathrm{e}^{\mathrm{i} \theta_{0}}}(s)$. Applying ergodicity, one may now show that $\tilde{f}_{s}$ tends to $f$ almost everywhere as $s \rightarrow 0$ non-tangentially. Hence results from one-dimensional theory can be used to deduce results on $H^{\infty}\left(\mathbb{T}^{\infty}\right)$. However, while in principle still feasible for $p \in[1, \infty)$, this approach becomes cumbersome since $F_{\mathrm{e}^{\mathrm{i} \theta}}(s)$ is only defined on the strip $0<\operatorname{Re} s \leq 1 / 2$ for almost every $\mathrm{e}^{\mathrm{i} \theta_{0}}$, and so the resulting Fatou-type statements are far from trivial.

One of the aims of this note is to describe an alternative approach to Fatou-type approximation 11 of certain functions on the polydisc, which is in the same spirit as the extensions mentioned above, but is easier to deal with and yields stronger results. Our idea is simple: given the function $f\left(\mathrm{e}^{\mathrm{i} \theta_{1}}, \mathrm{e}^{\mathrm{i} \theta_{2}}, \ldots\right)$, define a family of

\footnotetext{
${ }^{1}$ We are especially interested in the pointwise convergence at a boundary point since, by using the density of polynomials, practically any natural and well-defined radial approximation scheme leads to approximation in the $L^{p}$ norm for $p \in(1, \infty)$, and in the weak ${ }^{*}$ sense for $p=\infty$.
} 
functions

$$
f_{\xi}\left(\mathrm{e}^{\mathrm{i} \theta}\right):=f\left(\xi \mathrm{e}^{\mathrm{i} \theta_{1}}, \xi^{2} \mathrm{e}^{\mathrm{i} \theta_{2}}, \ldots\right), \quad \mathrm{e}^{\mathrm{i} \theta} \in \mathbb{T}^{\infty},
$$

where $\xi \in \mathbb{D}$ is a complex parameter from the unit disc. We will soon define $f_{\xi}$ in a precise manner, but one should note that $f_{\xi}\left(\mathrm{e}^{\mathrm{i} \theta}\right)$ is also well-defined pointwise by the mere fact that $\left(\xi \mathrm{e}^{\mathrm{i} \theta_{1}}, \xi^{2} \mathrm{e}^{\mathrm{i} \theta_{2}}, \ldots\right) \in \ell^{2}$ since $|\xi|<1$. The usefulness of introducing $f_{\xi}$ lies in the possibility of fixing $\mathrm{e}^{\mathrm{i} \theta} \in \mathbb{T}^{\infty}$ and employing, with a slight abuse of notation, the function of one variable $\xi \mapsto f_{\xi}\left(\mathrm{e}^{\mathrm{i} \theta}\right)$ in order to transfer one-dimensional tools to the infinite-dimensional situation. Note that $f_{\xi}\left(\mathrm{e}^{\mathrm{i} \theta}\right)$ is harmonic with respect to $\xi \in \mathbb{D}$ exactly when $f$ has Fourier spectrum supported on $\mathbb{N}_{0}^{\infty} \cup\left(-\mathbb{N}_{0}^{\infty}\right)$.

Let us begin by considering Poisson extensions of general measures. We define the space $\mathrm{Wi}\left(\mathbb{T}^{\infty}\right) \subset C\left(\mathbb{T}^{\infty}\right)$ consisting of the continuous functions $f: \mathbb{T}^{\infty} \rightarrow \mathbb{C}$ with absolutely convergent Fourier series, i.e., with $\sum_{\nu \in \mathbb{Z}_{0}^{\infty}}|\widehat{f}(\nu)|<\infty$. For any such $f$, the absolute convergence ensures that its unique polyharmonic extension to $\mathbb{D}^{\infty}$ is well-defined and actually continuous in all of the closure $\overline{\mathbb{D}}^{\infty}$. With yet a slight abuse of notation, we also denote this extension by $f$ so that for any $z=\left(\rho_{1} e^{i \theta_{1}}, \rho_{2} e^{i \theta_{2}} \ldots\right) \in \overline{\mathbb{D}}^{\infty}$, we have

$$
f(z)=\sum_{\nu \in \mathbb{Z}_{0}^{\infty}} \widehat{f}(\nu) \rho^{|\nu|} e^{i \nu \cdot \theta} .
$$

Here, we employ the abbreviations

$$
\rho^{|\nu|}:=\rho_{1}^{\left|\nu_{1}\right|} \rho_{2}^{\left|\nu_{2}\right|} \ldots \rho_{\ell}^{\left|\nu_{\ell}\right|} \text { and }|\nu|_{1}:=\sum_{j=1}^{\ell}\left|\nu_{j}\right|,
$$

for any multi-index $\nu=\left(\nu_{1}, \ldots, \nu_{\ell}\right) \in \mathbb{Z}_{0}^{\infty}$.

Let $\mu$ be finite Borel measure on $\mathbb{T}^{\infty}$, and set $\nu^{*}:=\left(\nu_{1}, 2 \nu_{2}, \ldots, \ell \nu_{\ell}\right)$. Then for any $\xi=r e^{i t} \in \overline{\mathbb{D}}$, we set, in accordance with (1),

$$
\mu_{\xi}=\sum_{\nu \in \mathbb{Z}_{0}^{\infty}} \widehat{\mu}(\nu) r^{\left|\nu^{*}\right|_{1}} e^{i t\left(\nu_{1}+2 \nu_{2}+\ldots\right)} e^{i \nu \cdot \theta} .
$$

Then, it holds that $\mu_{\xi} \in \mathrm{Wi}\left(\mathbb{T}^{\infty}\right)$ for all $\xi \in \mathbb{D}$, as one may compute

$$
\left\|\mu_{\xi}\right\|_{\mathrm{Wi}} \leq c \sum_{\nu \in \mathbb{Z}_{0}^{\infty}}|\xi|^{\left|\nu^{*}\right|_{1}}=c \prod_{j=1}^{\infty}\left(1+2 \sum_{k=1}^{\infty}|\xi|^{k j}\right)=c \prod_{j=1}^{\infty}\left(\frac{1+|\xi|^{j}}{1-|\xi|^{j}}\right)<\infty .
$$

We may then define a 'radial' maximal function of $\mu$ at every point $e^{i \theta} \in \mathbb{T}^{\infty}$ via

$$
M \mu\left(e^{i \theta}\right):=\sup _{r \in(0,1)}\left|\mu_{r}\left(e^{i \theta}\right)\right| \text {. }
$$

Remark 1. Observe that although it follows from the above argument that the function $\xi \mapsto \mu_{\xi}$ is continuous for every fixed $\mathrm{e}^{\mathrm{i} \theta} \in \mathbb{T}^{\infty}$, it is harmonic with respect to the variable $\xi \in \mathbb{D}$ if and only if the Fourier transform of $\mu$ has support on the set $\mathbb{N}_{0}^{\infty} \cup\left(-\mathbb{N}_{0}^{\infty}\right)$. 
e denote by $A_{m} \mu$ Bohr's ' $m$ te Abschnitt' of the measure $\mu$, which is defined by

$$
A_{m} \mu \sim \sum_{\eta \in \mathbb{Z}^{m}} \widehat{\mu}(\widetilde{\eta}) \mathrm{e}^{\mathrm{i} \theta \cdot \widetilde{\eta}}
$$

where $\widetilde{\eta}=\left(\eta_{1}, \ldots \eta_{m}, 0,0, \ldots\right)$ for $\eta \in \mathbb{Z}^{m}$. In other words, the harmonic extensions satisfy $A_{m} \mu(z)=\mu\left(z_{1}, \ldots, z_{m}, 0,0, \ldots\right)$ for any $z \in \ell^{1} \cap \mathbb{D}^{\infty}$.

\section{Theorem 1.}

(i) For any finite Borel measure $\mu$ on $\mathbb{T}^{\infty}$, one has

$$
\int_{\mathbb{T}^{\infty}}\left|\mu_{\xi}\left(\mathrm{e}^{\mathrm{i} \theta}\right)\right| \mathrm{d} \theta \leq\|\mu\|_{T V} \text { for all } \xi \in \mathbb{D} \quad \text { and } \quad \mu_{r} \stackrel{w^{*}}{\longrightarrow} \mu \quad \text { as } r \nearrow 1 .
$$

(ii) When restricted to measures with Fourier spectrum supported on $\mathbb{N}_{0}^{\infty} \cup\left(-\mathbb{N}_{0}^{\infty}\right)$, $M$ is of weak type 1-1, i.e., there is $C<\infty$ such that for any finite Borel measure $\mu$ on $\mathbb{T}^{\infty}$, with Fourier spectrum supported on $\mathbb{N}_{0}^{\infty} \cup\left(-\mathbb{N}_{0}^{\infty}\right)$, and $\lambda>0$, it holds that

$$
\left|\left\{\mathrm{e}^{\mathrm{i} \theta} \in \mathbb{T}^{\infty}: M \mu\left(\mathrm{e}^{\mathrm{i} \theta}\right)>\lambda\right\}\right| \leq \frac{C\|\mu\|_{\mathrm{TV}}}{\lambda} .
$$

Moreover, the finite radial limit $\mu^{*}\left(\mathrm{e}^{\mathrm{i} \theta}\right):=\lim _{r \rightarrow 1^{-}} \mu_{r}\left(\mathrm{e}^{\mathrm{i} \theta}\right)$ exists for almost every $\mathrm{e}^{\mathrm{i} \theta} \in \mathbb{T}^{\infty}$.

(iii) For any $f \in L^{1}\left(\mathbb{T}^{\infty}\right)$ with Fourier spectrum supported on $\mathbb{N}_{0}^{\infty} \cup\left(-\mathbb{N}_{0}^{\infty}\right)$, one has $f\left(\mathrm{e}^{\mathrm{i} \theta}\right)=\lim _{r \rightarrow 1} f_{r}\left(\mathrm{e}^{\mathrm{i} \theta}\right)$ for a.e. $\mathrm{e}^{\mathrm{i} \theta} \in \mathbb{T}^{\infty}$. Moreover, $\left\|f_{r}-f\right\|_{1} \rightarrow 0$ as $r \nearrow 1$.

(iv) There is $C<\infty$ such that for any $f \in H^{1}\left(\mathbb{T}^{\infty}\right)$,

$$
\|M f\|_{1} \leq C\|f\|_{H^{1}\left(\mathbb{T}^{\infty}\right)} .
$$

Similarly, 






$\square$

ㅁ

$\square$

$\square$ 
(b) The function

$$
u(z):=\prod_{n=1}^{\infty}\left(1+i \frac{z_{n}+\bar{z}_{n}}{2 n}\right)
$$

is in $L^{\infty}\left(\mathbb{T}^{\infty}\right)$ and fails at almost every boundary point to have radial boundary limit in the same sense as in (a).

The most interesting feature of these examples is that they allow us, in a simpler setting than in Theorem 4, to explain how to find a bad radial approach that is independent of the boundary point $\mathrm{e}^{\mathrm{i} \theta} \in \mathbb{T}^{\infty}$. As Theorem 4 (whose proof we give shortly) covers the phenomena displayed by both examples, we discuss only the main details of example (a).

To that end, we first note that, by the independence of the variables $\mathrm{e}^{\mathrm{i} \theta_{n}}$, the series

$$
\sum_{n=1}^{\infty} \frac{\operatorname{Re} \mathrm{e}^{\mathrm{i} \theta_{n}}}{n}=\sum_{n=1}^{\infty} \frac{\cos \left(\theta_{n}\right)}{n}
$$

is conditionally convergent almost everywhere on $\mathbb{T}^{\infty}$ (see, e.g., [7, Lemma 3.14, p. 46]). However, since the terms are bounded and $\sum_{n=1}^{\infty} \mathbf{E} \frac{\left|\cos \left(\theta_{n}\right)\right|}{n}=\infty$, we deduce from [7, Lemma 3.14, p. 46] that at almost every boundary point the series is not absolutely convergent. Hence for every $M \in \mathbb{N}$, as $N \rightarrow \infty$, we have

$$
\mathbf{P}\left(\sum_{n=M}^{N} \frac{\left|\cos \left(\theta_{n}\right)\right|}{n} \geq 1\right) \longrightarrow 1,
$$

and we may use the Borel-Cantelli lemma to inductively choose a sequence $m_{1}<$ $m_{2}<m_{3}<\cdots$ so that for almost every $\mathrm{e}^{\mathrm{i} \theta} \in \mathbb{T}^{\infty}$, and $k \geq k_{0}$ large enough, we have

$$
\sum_{m_{k}+1}^{m_{k+1}} \frac{\left|\cos \left(\theta_{n}\right)\right|}{n} \geq 1 \text {. }
$$

We now describe the bad approach working for almost every boundary point. For each $k$ we choose a vector $\mathbf{r}_{k}=\left(r_{k, 1}, r_{2, k}, \ldots\right)$ as follows. For $1 \leq k \leq 2^{m_{1}}$, we set

$$
\mathbf{r}_{k}=\left(r_{1, k}, \ldots, r_{m_{1}, k}, 0,0,0, \ldots\right)
$$

so that the first $m_{1}$ coordinates run through all $2^{m_{1}}$ different $m_{1}$-tuples consisting of 0 and $1 / 2$.

For the next $2^{m_{2}-m_{1}}$ indices $k$, we choose

$$
\mathbf{r}_{k}=(\underbrace{1-m_{1}^{-1}, \ldots, 1-m_{1}^{-1}}_{\text {first } m_{1} \text { entries }}, \underbrace{r_{m_{1}+1, k}, \ldots, r_{m_{2}, k}}_{\text {middle block }}, \underbrace{0,0,0, \ldots}_{\text {all zeroes }})
$$

so that the middle block can run through all tuples consisting of 0 and $1 / 2$.

Similarly, for the next $2^{m_{3}-m_{2}}$ coordinates, we choose

$$
\mathbf{r}_{k}=(\underbrace{1-m_{2}^{-1}, \ldots, 1-m_{2}^{-1}}_{\text {first } m_{2} \text { entries }}, \underbrace{r_{m_{2}+1, k}, \ldots, r_{m_{3}, k}}_{\text {middle block }}, \underbrace{0,0,0, \ldots}_{\text {all zeroes }}),
$$


again, so that the middle block can run through all tuples consisting of 0 and $1 / 2$.

If we continue in this way, it is clear that we get a sequence $\mathbf{r}_{k}$ such that, for fixed $n$, we have $r_{n, k} \nearrow 1^{-}$as $k \rightarrow \infty$. Moreover, given arbitrarily large $\ell \in \mathbb{N}$, for a suitable interval of indices $k$ it holds that

$$
\operatorname{Re} f\left(\mathbf{r}_{\mathbf{k}} \mathrm{e}^{\mathrm{i} \theta}\right)=\left(1-\frac{1}{m_{\ell}}\right) \sum_{n=1}^{m_{\ell}} \frac{\cos \left(\theta_{n}\right)}{n}+\sum_{n=m_{\ell}+1}^{m_{\ell+1}} r_{m_{\ell}, k} \frac{\cos \left(\theta_{n}\right)}{n}
$$

The first sum remains unchanged as $k$ varies in this interval, but the second term will oscillate between values close to 0 , and, in absolute value, larger than $1 / 2$. Since for almost every fixed boundary point this behaviour takes place infinitely many times, the statement follows.

We remark that the function in part (a) of the example is very close to being in $H^{\infty}\left(\mathbb{T}^{\infty}\right)$ in the sense that for some $c>0$ it holds that $\int_{\mathbb{T}_{\infty}} \exp \left(c\left|f\left(e^{i \theta}\right)\right|^{2}\right) \mathrm{d} \theta<$ $\infty$. The reason this holds is essentially that the Taylor series for this function is lacunary in a strong sense. Namely, the variables $z_{n}$ are independent of each other The argument for the function in part (b) is essentially a minor modification of the argument from the first example, although, initially it was inspired by the inductive method to construct Rudin-Shapiro polynomials. We mention that the uniform bound can be seen by writing

$$
|u(z)|=\sqrt{\prod_{n=1}^{\infty}\left(1+\frac{(z+\bar{z})^{2}}{4 n^{2}}\right)} .
$$

We now turn to the proof of Theorem 4 .

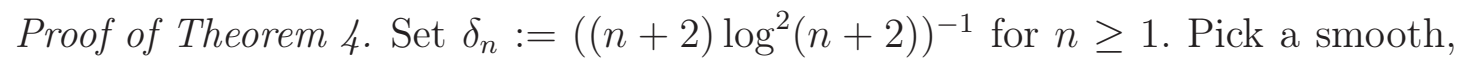
non-negative, and even function $\psi$ that satisfies $\psi(t)=1$ for $t \in(-1 / 4,1 / 4)$, $\psi(t)=0$ for $|t| \geq 1 / 2$ and $|\psi(t)| \leq 1$ for all $t$. We construct $f$ as the product

$$
f(z):=\prod_{n=1}^{\infty} f_{n}\left(z_{n}\right):=\prod_{n=1}^{\infty} \exp \left(-u_{n}\left(z_{n}\right)-\mathrm{i} \widetilde{u}_{n}\left(z_{n}\right)\right),
$$

where $u_{n}$ is the positive harmonic function on $\mathbb{D}$ with boundary values

$$
u_{n}\left(\mathrm{e}^{i t}\right):=\psi\left(t / \delta_{n}\right) \quad \text { for } \quad t \in[-\pi, \pi)
$$

One should note that the functions $f_{n}$ are continuous up to the boundary and real at the origin.

To see that the product converges, we may use the ' $m$ te Abschnitt' $A_{m} f(z)=$ $\prod_{n=1}^{m} f_{n}\left(z_{n}\right)$. From the definition, we obtain that the $A_{m} f(0)$ converges to a nonzero value since

$$
A_{m}(0)=\exp \left(-\sum_{n=1}^{m} \frac{1}{2 \pi} \int_{\mathbb{T}} u_{n}\left(\mathrm{e}^{i \theta_{n}}\right) \mathrm{d} \theta_{n}\right) \geq \exp \left(-\sum_{n=1}^{\infty} \delta_{n}\right)>0 .
$$

Hence, by a standard weak* convergence argument, $A_{m} f$ converges to a nontrivial element $f \in H^{\infty}\left(\mathbb{T}^{\infty}\right)$. This can also be seen following an argument of 
Hilbert which shows that $f$ has bounded point evaluations at all $z \in \mathbb{D}^{\infty} \cap c_{0}$ (see [8]). Moreover, by the Herglotz representation of $-\log f_{n}\left(z_{n}\right)$ and the fact that $\frac{1}{2 \pi} \int_{\mathbb{T}}\left|u_{n}\right| \sim \delta_{n}$, we deduce that at any point $\left(z_{1}, z_{2}, \ldots\right) \in \mathbb{D}^{\infty} \cap c_{0}$ it holds that

$$
|f(z)| \geq \exp \left(-C_{1} \sum_{n=1}^{\infty} \frac{\delta_{n}}{1-\left|z_{n}\right|}\right)
$$

so that $f$ is non-vanishing on $c_{0} \cap \mathbb{D}^{\infty}$.

In order to prove (i), we observe that by basic estimates for the Poisson kernel, the radial maximal function of $u_{n}$ satisfies $M u_{n}\left(e^{i t}\right) \geq C_{2} \min \left(1, \delta_{n}|t|^{-1}\right)$ for some constant $C_{2}>0$ and all $t \in[-\pi, \pi)$. It follows that

$$
\int_{\mathbb{T}} M u_{n} \frac{\mathrm{d} t}{2 \pi} \geq C_{2} \delta_{n} \log \left(1 / \delta_{n}\right) \geq \frac{C_{3}}{(n+2) \log (n+2)} .
$$

In particular, this yields

$$
\sum_{n=1}^{\infty} \int_{\mathbb{T}} M u_{n} \frac{\mathrm{d} t}{2 \pi}=\infty
$$

Since $0 \leq M u_{n} \leq 1$, we may use (14) and [7, Lemma 3.14, p. 46] to infer that

$$
\sum_{n=1}^{\infty} M u_{n}\left(\theta_{n}\right)=\infty \quad \text { for almost every }\left(\mathrm{e}^{i \theta_{n}}\right) \in \mathbb{T}^{\infty} .
$$

In other words, for almost every boundary point $\left(\mathrm{e}^{i \theta_{n}}\right) \in \mathbb{T}^{\infty}$ there are radii $r_{1}^{\prime}, r_{2}^{\prime}, \ldots<1$ such that

$$
\sum_{n=1}^{\infty} u_{n}\left(r_{n}^{\prime} \theta_{n}\right)=\infty
$$

We may especially choose an increasing sequence $\nu_{\ell}$ of indices so that

$$
\sum_{n=\nu_{\ell+1}}^{\nu_{\ell+1}} u_{n}\left(r_{n}^{\prime} \theta_{n}\right) \geq 4^{\ell} \quad \text { for all } \ell>1 .
$$

The desired radial approach for part (i) of the Theorem is obtained by choosing for this boundary point $r_{n, k}:=1$ for $n \leq \nu_{k}, r_{n, k}:=r_{n}^{\prime}$ for $\nu_{k}<n \leq \nu_{k+1}$, and $r_{n, k}:=0$ for $\nu_{k+1}<n$, and finally by slightly perturbing the chosen radii away from 1.

For part (ii) we perform basically the same argument as above, where the role of the maximal function $M u_{n}$ is taken by the absolute value of the conjugate function $\left|\widetilde{u}_{n}\right|$. Namely, by the definition of the conjugate function we see that $\left|\widetilde{u}_{n}\right|\left(e^{i t}\right) \geq$ $C_{4} \min \left(1, \delta_{n}|t|^{-1}\right)$ for some constant $C_{4}>0$, and for all $t \in[-\pi, \pi) \backslash\left[-2 \delta_{n}, 2 \delta_{n}\right]$. As before, it follows that $\int_{\mathbb{T}}\left|\widetilde{u}_{n}\right| \geq \delta_{n} \log \left(1 / \delta_{n}\right) \geq \frac{C_{5}}{(n+2) \log (n+2)}$ and we obtain the analogue of (14) for the functions $\left|\widetilde{u}_{n}\right|$. This together with the independence and the uniform boundedness of the functions $\widetilde{u}_{n}$ (recall that $\psi$ is smooth and the 
Hilbert transform is locally essentially scaling invariant) yields, for almost every boundary point,

$$
\sum_{n=1}^{\infty}\left|\widetilde{u}_{n}\left(i \theta_{n}\right)\right|=\infty .
$$

The proof is finished like in Example1, described above, and we obtain the desired radial approach where the fluctuations of $\arg f$ remain large. This would not yield the counterexample in points where $f\left(e^{i \theta}\right)=0$, but by Corollary 2 the measure of such points is zero.

The following questions appear quite interesting:

Question 1. Is it possible to remove the restriction on the Fourier spectrum in Theorem 1]?

Question 2. Does there exist a bounded analytic function $f \in H^{\infty}\left(\mathbb{T}^{\infty}\right)$ such that almost surely the radial convergence fails even if the approach is limited by assuming decreasing radii in $n$, i.e. $r_{n, k} \geq r_{n+1, k}$ for all $n, k$ ?

Question 3. What is the answer to the above question under the added condition that the radial approach does not depend on the point on the boundary?

Question 4. What can one say about non-tangential approach for functions in $H^{\infty}\left(\mathbb{T}^{\infty}\right)$, or more generally, in $H^{p}\left(\mathbb{T}^{\infty}\right)$ ?

Question 5. For which radial approaches is the brothers Riesz theorem on the uniqueness of boundary values true? That is, when is it true for all $f \in H^{\infty}\left(\mathbb{T}^{\infty}\right)$ that having vanishing radial boundary values on a set of positive Lebesgue measure implies $f \equiv 0$ ? Note that this is not true for all radial approaches as is demonstrated by Theorem 4 .

\section{ACKNOWLEDGEMENTS}

The authors would like to thank Michał Wojciechowski for pointing out an error in a previous version of the paper.

\section{REFERENCES}

1. Harald Bohr, Über die Bedeutung der Potenzreihen unendlich vieler Variabeln in der Theorie der Dirichletschen Reihen $\sum a_{n} / n^{s}$, Nachr. Akad. Wiss. Göttingen Math.-Phys. Kl. (1913), 441-488.

2. Brian J. Cole and T. W. Gamelin, Representing measures and Hardy spaces for the infinite polydisk algebra, Proc. London Math. Soc. 53 (1986), no. 3, 112-142.

3. John B. Garnett, Bounded Analytic Functions, Academic Press, 1981.

4. Henry Helson, Compact groups and Dirichlet series, Ark. Mat. 8 (1969), 139-143.

5. Henry Helson and David Lowdenslager, Prediction theory and Fourier series in several variables, Acta Math. 99 (1958), 165-202.

6. David Hilbert, Wesen und Ziele einer Analysis der unendlichvielen unabhängigen Variabeln., Rend. Circ. Mat. Palermo 27 (1909), 59-74. 
7. Olav Kallenberg, Foundations of modern probability, second ed., Probability and its Applications (New York), Springer-Verlag, New York, 2002.

8. Håkan Hedenmalm, Peter Lindqvist, and Kristian Seip, A Hilbert space of Dirichlet series and systems of dilated functions in $L^{2}(0,1)$, Duke Math. J. 86 (1997), 1-37.

9. Hervé Queffélec and Martine Queffélec, Diophantine approximation and Dirichlet series, Harish-Chandra Research Institute Lecture Notes, vol. 2, Hindustan Book Agency, New Delhi, 2013.

10. Walter Rudin, Fourier Analysis on Groups, Interscience Publishers (a division of John Wiley and Sons), New York-London, 1962.

11. Walter Rudin, Function theory in polydiscs, W. A. Benjamin, Inc., New York-Amsterdam, 1969.

12. Eero Saksman and Kristian Seip, Integral means and boundary limits of Dirichlet series, Bull. Lond. Math. Soc. 41 (2009), no. 3, 411-422.

13. Anthony Zygmund, Trigonometric series: Vols. I, II, Second edition, reprinted with corrections and some additions, Cambridge University Press, London, 1968.

Centre for Mathematical Sciences, Lund University, P.O. Box 118, SE-221 00 LUND, SWEDEN

E-mail address: aleman@maths.1th.se

Centre for Mathematical Sciences, Lund University, P.O. Box 118, SE-221 00 LUND, SwEDEN

E-mail address: janfreol@maths.1th.se

Department of Mathematics and Statistics, University of Helsinki, PO Box 68, FI-00014 Helsinki, Finland

E-mail address: eero.saksman@helsinki.fi 